\title{
Consideration of Neutral Beam Prompt Loss in the Design of a Tokamak Helicon Antenna
}

\author{
D.C. Pace, M.A. Van Zeeland, B. Fishler, C. Murphy \\ General Atomics, P.O. Box 85608, San Diego, CA 92186-5608, USA
}

\begin{abstract}
Neutral beam prompt losses (injected neutrals that ionize such that their first poloidal transit intersects with the wall) can put appreciable power on the outer wall of tokamaks, and this power may damage the wall or other internal components. These prompt losses are simulated including a protruding helicon antenna installation in the DIII-D tokamak and it is determined that $160 \mathrm{~kW}$ of power will impact the antenna during the injection of a particular neutral beam. Protective graphite tiles are designed in response to this modeling and the wall shape of the installed antenna is precisely measured to improve the accuracy of these calculations. Initial experiments confirm that the antenna component temperature increases according to the amount of neutral beam energy injected into the plasma. In this case, only injection of beams that are aimed counter to the plasma current produce an appreciable power load on the outer wall, suggesting that the effect is of little concern for tokamaks featuring only co-current neutral beam injection. Incorporating neutral beam prompt loss considerations into the design of this in-vessel component serves to ensure that adequate protection or cooling is provided.
\end{abstract}

Keywords: neutral beam, prompt loss, helicon antenna, thermal design

Email address: pacedc@fusion.gat.com (D.C. Pace)

Preprint submitted to Fusion Engineering and Design

July 20, 2016

(C) 2016. This manuscript version is made available under the Elsevier user license http://www.elsevier.com/open-access/userlicense/1.0/ 


\section{Introduction}

A variety of plasma-facing components are challenged to survive the intense radiation and thermal energy environment of the magnetically confined plasmas produced in tokamaks. These range from simple diagnostics to major systems

5 that provide auxiliary heating such as wave injectors. For a potentially large component such as a high power antenna, the proximity to the plasma encourages design that accounts for the nearby magnetic field [1] and rewards such considerations with improved plasma performance [2]. Assessments of potential energetic ion (either charged fusion products or those produced from beam and/or wave heating) losses are separately critical for ITER-like devices because these losses may both reduce the effective plasma heating from neutral beams and damage wall components [3]. Energetic ion orbits are considerably larger than those of the thermal plasma particles, and this can require unique designs for protecting plasma-facing components.

Neutral beams are capable of injecting up to $20 \mathrm{MW}$ of power into the DIIID tokamak [4, 5. Some of the injected neutrals ionize in the plasma such that their resulting orbit takes them directly into a plasma-facing surface. Those prompt loss beam ions can result in $\mathcal{O}(1) \mathrm{MW} / \mathrm{m}^{2}$ power loads on the wall. Such power loads were considered during the design of a helicon wave antenna that is proud of the standard wall surface. Analysis of the prompt losses from the neutral beams is used to aid in the design of the helicon antenna, including the design of protective tiles surrounding it.

The value of the helicon antenna is that its injected power is predicted to provide efficient off-axis current drive [6]. Injected from the outer wall, helicon waves will deposit their energy into the plasma before reaching the center. That desirable effect results in off-axis current drive that is an important component in experiments to develop steady state plasma scenarios. The reliability of wave driven off-axis current may be preferable to that sourced from the neutral beams themselves (producing a suitable off-axis neutral beam source is a considerable task [7]). Coupling helicon waves to the plasma requires propagating the wave 
across the spatial region between the magnetically confined plasma and the antenna. The smaller the distance between the helicon antenna and the confined plasma boundary, the better the injected waves will couple. Efforts to minimize this distance lead to a situation in which the power reaching the helicon antenna 35 due to neutral beam prompt losses is significant.

\section{Prototype Modeling}

DIII-D features a near-continuous wall with cut-outs for diagnostics and access for auxiliary heating. The plasma-facing tiles are composed of graphite. Figure 1(a) is an engineering drawing cross-section of DIII-D showing a typi-

40 cal plasma (major radius $R_{\text {major }}=1.7 \mathrm{~m}$ and minor radius $a=0.7 \mathrm{~m}$ ) and highlighting the region identified for helicon antenna installation. This antenna installation location was determined based on the availability of diagnostic port access for connection feedthroughs and the existence of a wide viewing region for an infrared camera diagnostic [8]. Predetermining the location of the antenna installation greatly simplified the prompt loss calculations because the new wall shape that incorporates the antenna profile had been set. A photograph of the late-stage installation is given in Fig. 1(b). The center of the antenna is installed at $\phi_{\text {DIII-D }}=195^{\circ}$ and the ports just above it and below it are labeled $195 \mathrm{R}+1$ and 195R0, respectively (the naming scheme indicates the toroidal angle location and the fact that these are $[R]$ adial ports located above the midplane $[+1]$ and at the midplane $[0])$.

Eight distinct neutral beams in DIII-D are each capable of injecting up to 2.5 MW into the plasma. These beams are named according to their toroidal angle location and whether they exist in the left or right side of the housing (two beams per housing, e.g., $210 \mathrm{~L}$ is located at $\phi_{\text {DIII-D }}=210^{\circ}$ in the left side of the housing). Prompt losses are determined based on modeling of the neutral deposition and the resulting ion trajectories. The deposition (i.e., the ionization profile) is calculated using a Monte Carlo method that includes the three-dimensional geometry of the beams and the atomic cross-section data for 
ionization probabilities from ADAS [9, 10. Radial profiles of plasma parameters are provided as measured (e.g., magnetic equilibria from EFIT reconstructions [1], ion density and temperature from charge exchange recombination spectroscopy [12], and electron density and temperature from Thomson scattering [13]). This provides a data set of ions specifying their full location and velocity vector, and that data is used as the initialization for a gyrocenter calculation of their trajectory. Deposition results from this modeling are shown in Fig. 2 for shot $158527\left(B_{t}=1.25 \mathrm{~T}\right.$ and $\left.I_{p}=0.7 \mathrm{MA}\right)$. Figures 2(a,b) show the deposition as a projection in the $R z$-plane. One important capability of the deposition code is that it includes ionization in the scrape-off layer, which, even though the number of neutrals ionizing outside the confined plasma is generally small, has still been found to be a contributing factor in measurements of lost beam ions [14. The solid white line in Fig. 2(b) is the separatrix and any deposition outside of this represents scrape-off layer ionization. Figures $2(\mathrm{c}, \mathrm{d})$ provide a top-view of the deposition to highlight the distinct toroidal dependence. While this deposition is calculated in three dimensions, the magnetic equilibrium and plasma profiles are all input as axisymmetric properties.

The deposition profile provides the initialization information necessary to calculate the resulting beam ion orbit: initial position $\left(R_{\text {major }}, \phi, z\right)$, and initial velocity vector $\left(v_{R}, v_{\phi}, v_{z}\right)$ known from the injection vector of the neutral. The so magnetic equilibrium is known separately. Example orbits from Fig. 3 serve to describe the matter at hand, namely that some injected neutrals ionize such that their orbit very quickly connects to the outer wall. Figure 3 includes two neutrals that ionize with nearly the same properties except that one begins with a velocity vector parallel to the plasma current (black trace) and the other begins with a velocity anti-parallel to the plasma current (red trace). These two arbitrary example orbits show that counter- $I_{p}$ injected neutrals feature orbits that begin on the inner leg of their banana trajectory whereas co- $I_{p}$ injected neutrals begin on the outer leg. Counter-injected neutral beams are therefore capable of losing considerably more ions to the outer wall than a similarly built beam that 90 injects along the direction of the plasma current. Whether an injected neutral 
eventually strikes the helicon antenna is dependent on the plasma parameters and the beam geometry. A wide range of plasma and beam conditions are modeled in order to address the scenarios most likely to place appreciable prompt loss power on the antenna.

The ion orbits are calculated using a gyrocenter code [15] that generally completes a 120,000 orbit calculation in under 2 hours. A fast completion time is necessary in order to model many different scenarios. A full orbit calculation that follows the position of the ion instead of its gyrocenter would be more than an order of magnitude slower without providing any significant advantage over the gyrocenter option. Full orbit results would provide better accuracy across the face of the helicon antenna, but that level of detail is not required to resolve questions such as whether an antenna component is in danger of melting due to power loading from neutral beam prompt losses. The value of the improved spatial accuracy from a full orbit calculation is also decreased without the ability to provide a similarly resolved wall shape, which is not available.

While ions are followed throughout the scrape-off layer, there is no account for neutralization due to charge exchange between the ions and neutrals. Depending on the neutral density, the fraction of beam ions that neutralize in the scrape-off layer can be significant. The consequence of neutralization is that it converts the ion to an unconfined neutral that will travel along whatever velocity vector the ion had at the moment of neutralization. Given the gyro-orbit of an ion in the tokamak magnetic field, this neutralization process can result in $50 \%$ of the beam ions passing back into the confined plasma. By ignoring this effect in the prompt loss power calculations here, we ensure that we calculate the largest possible amount of prompt loss power reaching the helicon antenna, and that increases the likelihood that an appropriate amount of shielding is designed (acknowledging that some events such disruptions could potentially produce a larger power load).

The modeling process for the helicon project includes pre-installation modeling using a prototype shape and then new calculations performed after determination of the actual antenna protrusion. Projections of the prototype and 
actual helicon limiter shape are shown in Fig. 4 along with the typical limiter shape away from the helicon antenna. Details of the final shape are given in Sec. 3. The prototype limiter shape appears very different from the final shape because it does not include protection tiles that were added following the modeling discussed below. The final limiter also extends up to $1.2 \mathrm{~cm}$ further into the vacuum chamber than the prototype. Both wall shapes are modeled with a toroidal extent of $186^{\circ} \leq \phi_{\text {DIII-D }} \leq 204^{\circ}$. Outside of this toroidal region the limiter shape is appropriately defined according to whether there is a port opening or solid tile coverage.

Modeling of the prompt losses striking the proposed helicon antenna installation was conducted using a plasma shape considered likely to provide one of the largest possible neutral beam prompt loss heat loads. Shot 158527 at $t=3000$ $\mathrm{ms}$ is a shape commonly used for advanced scenario experiments. The toroidal magnetic field, $B_{t}$, is reversed (counter-clockwise when viewing the tokamak from above) compared to standard DIII-D experiments. A reversed toroidal magnetic field means that the drift due to the gradient in the magnetic field, set according to the direction $\vec{B} \times \nabla \vec{B}$, is directed upward. Lost ions typically strike the wall at a vertical position determined according to this drift direction, and this means that reversed- $B_{t}$ plasmas cause the largest number of prompt loss ions to hit the helicon antenna in its position above the midplane. In general, antennas that are installed off midplane could be placed opposite of the expected vertical segment of beam ion prompt loss, but that is not possible for DIII-D since experiments utilizing both directions of toroidal magnetic field are commonplace. While there are simulations that include all of the neutral beams, it quickly became apparent that the counter- $I_{p}$ beam results in an order of magnitude larger heat load on the helicon antenna. Later modeling therefore only includes counter- $I_{p}$ neutral beams.

A summary of the results from one such simulation is shown in Fig. 5. This simulation follows 120,000 ionized particles and identifies those that hit the wall. The $R z$-projection of prompt losses from the $210 \mathrm{~L}$, counter- $I_{p}$, neutral beam is shown in Fig. 5(a). The wall shape shows the prototype helicon antenna protru- 
sion near $R_{\text {major }}=2.3 \mathrm{~m}$ and $z=0.3 \mathrm{~m}$. Separation between the ion location and the wall shape indicates the gyroradius of the ions in this gyrocenter orbit calculation. Figure 5(b) shows the projection (as a two-dimensional histogram) of prompt losses in the $\phi_{\text {DIII-D }} \theta$-plane, where $\theta$ is the poloidal angle. The edge of the helicon antenna appears as a sharp right-angle turn ending at approximately $\phi_{\text {DIII-D }}=200^{\circ}$ in this panel.

Prompt loss results are converted to power reaching the antenna based on the fractional number reaching the antenna and the total amount of power known to be injected by that beam. Continuing with the results shown in Fig. 5, the $210 \mathrm{~L}$ beam is simulated with an injection energy of $V_{\text {accel }}=80 \mathrm{kV}$ corresponding to an injected power (i.e., beam power reaching the plasma) of $P=2.6 \mathrm{MW}$. Of the 2.6 MW reaching the plasma, we estimate that $76 \%$ of that power is contained in the full energy component [4]. The lower energy components are ignored because they represent a smaller fraction of the power possibly reaching the helicon antenna, and since they also feature smaller gyroradii they are more easily blocked by protection tiles. This leaves the full energy power at 2.0 MW, and then the deposition calculation shows that $13 \%$ of the injected full energy neutrals are lost to shine-through and simply strike the opposite wall without ionizing. It should be noted that this shine-through level is large because this particular beam is aimed more perpendicularly to the background magnetic field and it therefore reaches the center post while the tangential beams are aimed across the tokamak, missing the center post, and feature shine-through percentages closer to zero. The total power from the full energy component of the beam injection is therefore 1.7 MW. The prompt loss calculation shows that $28.6 \%$ of this $1.7 \mathrm{MW}(0.5 \mathrm{MW})$ reaches the wall as prompt losses. Of this $0.5 \mathrm{MW}$ reaching the wall, $32 \%$ is identified as striking the antenna location. Taking this into account, $0.16 \mathrm{MW}$ is identified as hitting the helicon antenna. From the geometry of an ion gyrating about the background magnetic field it is possible to further separate the power deposition to determine that it is nearly evenly spread across the top of the antenna and the front (i.e., larger $\phi_{\text {DIII-D }}$ end). These results inform the design of protection tiles for the helicon antenna. 


\section{Modeling and Experience Following Installation}

185

The final installation location of the helicon antenna was measured with a coordinate measuring machine $(\mathrm{CMM})$. The CMM is installed in the DIII-D vacuum vessel and provides three-dimensional position data that is accurate to better than $0.5 \mathrm{~mm}$. A set of landmarks in the inconel vacuum vessel provide a reference for the CMM. Figure 6(a) shows a photograph of the helicon antenna protection tiles that are labeled according to their identification within the CMM data set. The final CMM survey includes 100 point measurements distributed across each of the 13 antenna tiles. Preference was given to measurement locations near the edges and innermost portions of the tiles in order to determine the areas of the entire helicon antenna structure that are closest to the plasma. In addition, the Faraday rods (these serve as electromagnetic shields placed just above the modules) from the modules on either end were surveyed, as was the single Langmuir probe tip [indicated in Fig. 6(a)]. A plot of all the CMM data is shown in Fig. 6(b). The full data set is then reduced to define a new limiter shape and this new shape is used in modeling of neutral beam prompt losses and comparison with experiments.

A first comparison is made between the actual antenna wall shape and the prototype as shown in Fig. 5. The deposition profile changes an insignificant amount consistent with variability in the Monte Carlo method. The fraction of prompt loss reaching the antenna increases from $32 \%$ in the prototype case to $42 \%$ using the actual wall shape. Total power deposited on the antenna therefore rises from $0.16 \mathrm{MW}$ to $0.20 \mathrm{MW}$, with the majority of this power striking the protective tiles. Calculating an increased power deposition with the actual wall shape from the helicon antenna is expected since the actual shape includes additional protection tiles and extends $1.2 \mathrm{~cm}$ further into the vacuum chamber (see Fig. 4).

After installing the helicon antenna it was possible to conduct plasma experiments, measure the temperature of the antenna, and then simulate the neutral beam prompt loss power for comparison. An initial plasma experiment 
is conducted with a magnetic field of $B_{t}=2.06 \mathrm{~T}$ and line-averaged density $3 \leq \bar{n}_{e} \leq 5 \times 10^{13} \mathrm{~cm}^{-3}$ with a plasma current ramp from 0.8 to $0.6 \mathrm{MA}$. The purpose of the current ramp is that it changes the radial orbit width (i.e., the banana width) of the beam ions, thereby allowing for a study of the power density dependence on $I_{p}$. Figure 7(a) shows the results from a simulation of 210L prompt loss reaching the antenna for the central value of $I_{p}=0.7 \mathrm{MA}$. 220 The ion orbit calculations are gyrocenter, and this post-processing considers full orbit effects by binning the ions across a $5^{\circ}$ toroidal extent preferentially directed toward lower toroidal angle (preference set according to the plasma current direction). The toroidal extent is determined based on a typical range of toroidal transit distances for the beam ions [16. The results indicate that the 225 higher toroidal angle edge helicon modules experience approximately $1 \mathrm{MW} / \mathrm{m}^{2}$ power density and the nearby protective tiles experience twice this value. Figure 7(b) shows the temperatures of the molybdenum Faraday rods in a SolidWorks [17. simulation applying this prompt loss power density for a duration of two seconds. In this case the Faraday rods reach a $762 \mathrm{C}$ temperature. A large difference in temperature between the toroidally separated modules is expected since the prompt loss heating pattern dominates on the higher toroidal angle side of the helicon antenna. Recognizing this toroidal dependence (and noting that it would switch sides during experiments in which the plasma current direction is reversed from standard), the shielding tiles of the antenna are ramped 235 [see Fig. 6(a)] such that some beam ions strike them instead of the Faraday rods or the copper modules. A ramp along the toroidal direction is the primary adjustment to the protective tiles in order to reduce the beam ion power flux reaching the more sensitive components of the helicon antenna. The gyroradii of beam ions in DIII-D is typically $4-5 \mathrm{~cm}$, meaning that their orbits will wrap around any tile protection extended radially beyond the helicon antenna (the emitted helicon wave field evanesces within a few $\mathrm{mm}$, so the protective tiles cannot protrude further than this or they will prevent the helicon waves from reaching the confined plasma).

A comparison of the measured and simulated module temperatures from 
shot 164468 is given in Fig. 8. The measured temperature is recorded by a thermocouple embedded in the module at the highest toroidal angle [see the Thermocouple \#7 label in Figs. 1(b)]. The absolute value of the time axis is arbitrary and extended in order to show the long term evolution of the helicon module temperature (the actual plasma exists for a duration of 5.4 seconds beginning near $t=30 \mathrm{~s}$ ). The simulated power load from the $210 \mathrm{~L}$ beam prompt loss is taken from Fig. 7(a) and the calculated temperature behavior shows both a faster rise and a higher peak temperature compared to the measured values. This result is not inconsistent considering that simulation parameters are chosen to ensure that simulated power loads are slightly larger than those actually produced during operation. The thermal conduction path to the thermocouple in the simulation is shorter than that actually installed in the real antenna (i.e., the actual tokamak remains more complex than the engineering model), which is also consistent with the differences between the simulated and measured temperature evolution.

260 A summary of the dependence of the measured helicon module temperature (thermocouple \#7) as a function of the 210L neutral beam injected energy is given in Fig. 9. This data is collected over a range of shots during the first day of plasma experiments intended to test the coupling of the antenna with the plasma. The peak rise in the temperature is shown instead of the absolute temperature to account for a variable starting temperature from plasma shots occurring earlier in the day. The 210L injected energy is totaled across the entire shot. For injected energies below 1 MJ there is no noticeable increase in helicon module temperature. Above 3.5 MJ of injected energy, however, the temperature rise becomes greater than $30 \mathrm{C}$ and the potential for damage in a single plasma shot is increased since a $30 \mathrm{C}$ rise in the bulk temperature corresponds to much higher surface temperature on the Faraday shield. This operational experience is used to aid in experiment planning by requiring extra precaution for proposed shots that will inject more than $3 \mathrm{MJ}$ of energy from the $210 \mathrm{~L}$ neutral beam.

Additional qualitative insights are uncovered from this modeling and the 
ensuing experiments. In both the simulations and the experiment, the power reaching the helicon antenna decreased as the plasma current increases. This general outcome is expected because the ion banana orbit width decreases as plasma current increases, meaning that fewer orbits are expected to reach the outer wall. Figure 10 shows the results of a set of simulations of the prompt loss from beam 210L in shot 164468 at three different plasma current values. Both the entire prompt loss power (black trace) and the power striking the helicon antenna (red trace) decrease as plasma current increases. Empirically, shots taken with plasma current above 0.8 MA were not observed to produce significant helicon antenna temperatures during beam injection. This is consistent with the specific simulation results of Fig. 10, but it is noted there may exist a plasma shape for which the prompt loss pattern changes significantly (e.g., smaller outer gaps).

\section{Conclusions}

Simulations of neutral beam prompt losses are used to inform the powerhandling needs of a new helicon antenna installation in the DIII-D tokamak. The antenna is expected to achieve optimal function if placed as closely as possible to the plasma, though within reason considering its need to survive plasma interaction at this minimal separation. A prototype antenna design was modeled and prompt loss power densities upwards of $1 \mathrm{MW} / \mathrm{m}^{2}$ were identified. Graphite protection tiles were designed based on this information with the goal of ensuring a safe existence for the copper modules. All of these aspects of the design are completely unrelated to actual operation of the helicon antenna, for the potentially damaging counter-current neutral beams operate across a wide range of DIII-D experiments that do not use the helicon antenna.

Following installation, new simulations are performed based on the measured antenna wall profile and the results are compared with engineering models of the temperature response of the antenna components. As predicted, the prompt loss power striking the antenna due to counter-current injection of the $210 \mathrm{~L}$ neutral 


\section{Acknowledgements}

The Authors wish to express their gratitude to K. Burrell, J. deGrassie, M. Fenstermacher, A. Kellman, R. Pinsker, R. Prater, and F. Turco for providing appropriate conditions to simulate. Effective beam stopping cross-sections Data and Analysis Structure (ADAS) compilation. The originating developer of ADAS is the JET Joint Undertaking. This work supported by the US Department of Energy, Office of Science, Office of Fusion Energy Sciences, using the DIII-D National Fusion Facility, a DOE Office of Science user facility, under Award DE-FC02-04ER54698.

\section{References}

[1] M. Garrett, S. Wukitch, Mitigation of radio frequency sheaths through magnetic field-aligned ICRF antenna design, Fus. Eng. Des. 87 (9) (2012)

प्र 1570 - 1575. doi:http://dx.doi.org/10.1016/j.fusengdes.2012.04.

325 011 .

URL http://www.sciencedirect.com/science/article/pii/ S0920379612002621

[2] S. J. Wukitch, M. L. Garrett, R. Ochoukov, J. L. Terry, A. Hubbard, B. Labombard, C. Lau, Y. Lin, B. Lipschultz, D. Miller, M. L. Reinke, ${ }_{330}$ D. Whyte, Alcator C-Mod Team, Characterization and performance of a field aligned ion cyclotron range of frequency antenna in Alcator C-Mod, 
335

Phys. Plasmas 20 (5) (2013) 056117. doi:http://dx.doi.org/10.1063/ 1.4803882 .

URL http://scitation.aip.org/content/aip/journal/pop/20/5/10. $1063 / 1.4803882$

[3] Jari Varje, Otto Asunta, Mario Cavinato, Mario Gagliardi, Eero Hirvijoki, Tuomas Koskela, Taina Kurki-Suonio, Yueqiang Liu, Vassili Parail, Gabriella Saibene, Seppo Sipilä, Antti Snicker, Konsta Särkimäki, Simppa Äkäslompolo, "Effect of plasma response on the fast ion losses due to ELM control coils in ITER," Nucl. Fusion (accepted 2016)

[4] J. Luxon, A design retrospective of the DIII-D tokamak, Nucl. Fusion 42 (5) (2002) 614 .

URL http://stacks. iop.org/0029-5515/42/i=5/a=313

[5] R. Buttery, the DIII-D Team, DIII-D research to address key challenges for ITER and fusion energy, Nucl. Fusion 55 (10) (2015) 104017.

URL http://stacks . iop.org/0029-5515/55/i=10/a=104017

[6] R. Prater, C. Moeller, R. Pinsker, M. Porkolab, O. Meneghini, V. Vdovin, Application of very high harmonic fast waves for off-axis current drive in the DIII-D and FNSF-AT tokamaks, Nucl. Fusion 54 (8) (2014) 083024.

$$
\text { URL http: //stacks . iop.org/0029-5515/54/i=8/a=083024 }
$$

[7] C. Murphy, M. Abrahim, P. Anderson, H. Chiu, H. Grunloh, M. Hansink, K. Holtrop, R.-M. Hong, A. Kellman, D. Kellman, P. Mauzey, S. Noraky, C. Pawley, J. Rauch, J. Scoville, M. Van Zeeland, H. Yip, R. Wood, M. Murakami, J. Park, W. Heidbrink, Overview of DIII-D off-axis neutral beam project, in: Fusion Engineering (SOFE), 2011 IEEE/NPSS 24th Symposium on, 2011, pp. 1-6. doi:10.1109/SOFE.2011.6052323

[8] C. J. Lasnier, S. L. Allen, R. E. Ellis, M. E. Fenstermacher, A. G. McLean, W. H. Meyer, K. Morris, L. G. Seppala, K. Crabtree, M. A.

घ Van Zeeland, Wide-angle ITER-prototype tangential infrared and visible viewing system for DIII-D, Rev. Sci. Instrum. 85 (11) (2014) -. 
doi:http://dx.doi.org/10.1063/1.4892897

URL http://scitation.aip.org/content/aip/journal/rsi/85/11/ $10.1063 / 1.4892897$

[9] M. A. Van Zeeland, N. M. Ferraro, W. W. Heidbrink, G. J. Kramer, D. C. Pace, X. Chen, T. E. Evans, R. K. Fisher, M. García-Muñoz, J. M. Hanson,

M. J. Lanctot, L. L. Lao, R. A. Moyer, R. Nazikian, D. M. Orlov, Modulation of prompt fast-ion loss by applied $n=2$ fields in the DIII-D tokamak, Plasma Phys. Control. Fusion 56 (1) (2014) 015009.

URL http://stacks .iop.org/0741-3335/56/i=1/a=015009

[10] Atomic Data and Analysis Structure (ADAS) compilation. ADAS http://adas.phys.strath.ac.uk.

[11] L. Lao, H. S. John, R. Stambaugh, A. Kellman, and W. Pfeiffer, Nucl. Fusion 25, 1611 (1985).

[12] K. H. Burrell, P. Gohil, R. J. Groebner, D. H. Kaplan, J. I. Robinson, and W. M. Solomon, Rev. Sci. Instrum. 75, 3455 (2004).

[13] T. N. Carlstrom, G. L. Campbell, J. C. DeBoo, R. Evanko, J. Evans, C. M. Greenfield, J. Haskovec, C. L. Hsieh, E. McKee, R. T. Snider, R. Stockdale, P. K. Trost and M. P. Thomas, Rev. Sci. Instrum. 63, 4901 (1992).

[14] X. Chen, W. Heidbrink, G. Kramer, M. V. Zeeland, M. Austin, R. Fisher,

R. Nazikian, D. Pace, C. Petty, Prompt non-resonant neutral beam-ion loss induced by Alfvén eigenmodes in the DIII-D tokamak, Nucl. Fusion 53,123019 (2013).

URL http://stacks . iop.org/0029-5515/53/i=12/a=123019

[15] M. A. Van Zeeland, W. W. Heidbrink, R. K. Fisher, M. García-Muñoz, G. J. Kramer, D. C. Pace, R. B. White, S. Aekaeslompolo, M. E. Austin, J. E. Boom, I. G. J. Classen, S. da Graça, B. Geiger, M. Gorelenkova, N. N. Gorelenkov, A. W. Hyatt, N. Luhmann, M. Maraschek, G. R. McKee, R. A. Moyer, C. M. Muscatello, R. Nazikian, H. Park, S. Sharapov, 
W. Suttrop, G. Tardini, B. J. Tobias, Y. B. Zhu, DIII-D, ASDEX Upgrade

Teams, Measurements and modeling of Alfvén eigenmode induced fast ion transport and loss in DIII-D and ASDEX Upgrade, Phys. Plasmas 18 (5)

(2011) 056114. doi:10.1063/1.3574663.

URL http://link.aip.org/link/?PHP/18/056114/1

[16] D. C. Pace, R. S. Granetz, R. Vieira, A. Bader, J. Bosco, D. S. Darrow, C. Fiore, J. Irby, R. R. Parker, W. Parkin, M. L. Reinke, J. L. Terry, S. M. Wolfe, S. J. Wukitch, S. J. Zweben, Energetic ion loss detector on

口 the Alcator C-Mod tokamak, Rev. Sci. Instrum. 83 (7) (2012) 073501. doi: $10.1063 / 1.4731655$

URL http://link . aip.org/link/?RSI/83/073501/1

${ }_{400}[17]$ http://www.solidworks.com. 

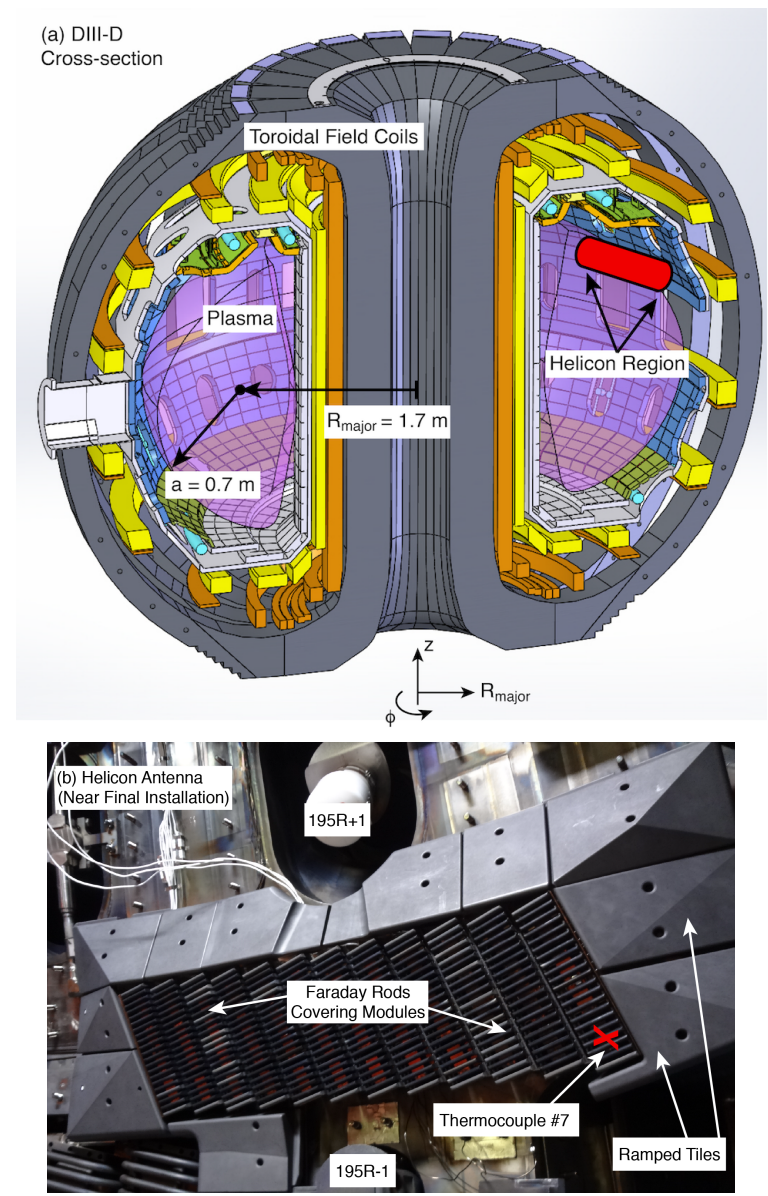

Figure 1: (a) Engineering drawing of the DIII-D tokamak with a highlight of the helicon antenna installation location slightly above the midplane. (b) Photograph of the helicon antenna as the installation process nears completion with the modules covered by Faraday shields (rods made from titanium-zirconium-molybdenum [TZM] coated with $\mathrm{B}_{4} \mathrm{C}$ ). 

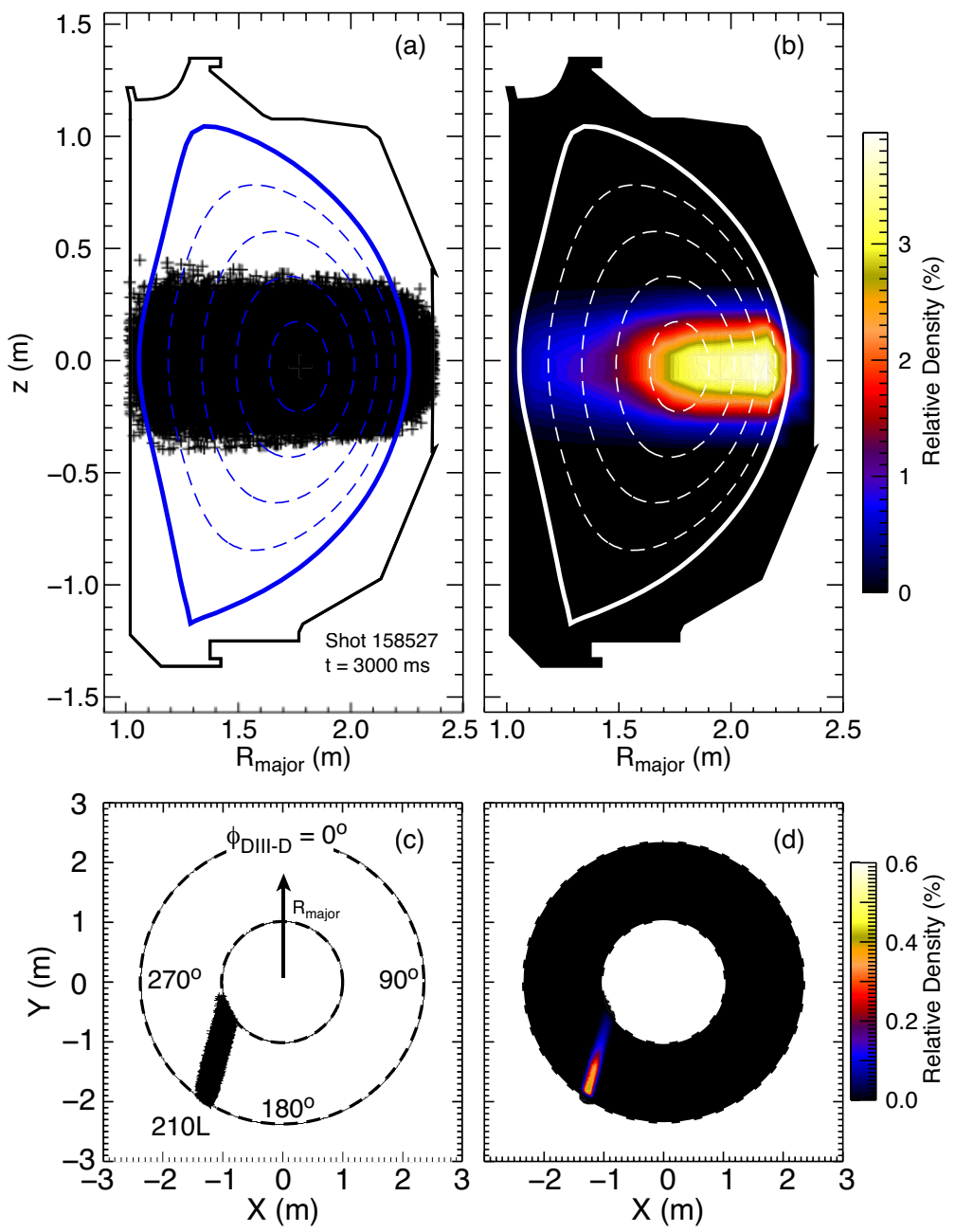

Figure 2: Modeled deposition profile of DIII-D neutral beam 210L at $t=3000 \mathrm{~ms}$ in shot 158527. The $R z$-projection of 120,000 neutrals is shown as (a) individual particles and (b) a contour highlighting the relative density (the total of all the elements in this display equal $100 \%$ ) across the plasma. Top views of this data are also provided with (c) individual markers and (d) density contour. 


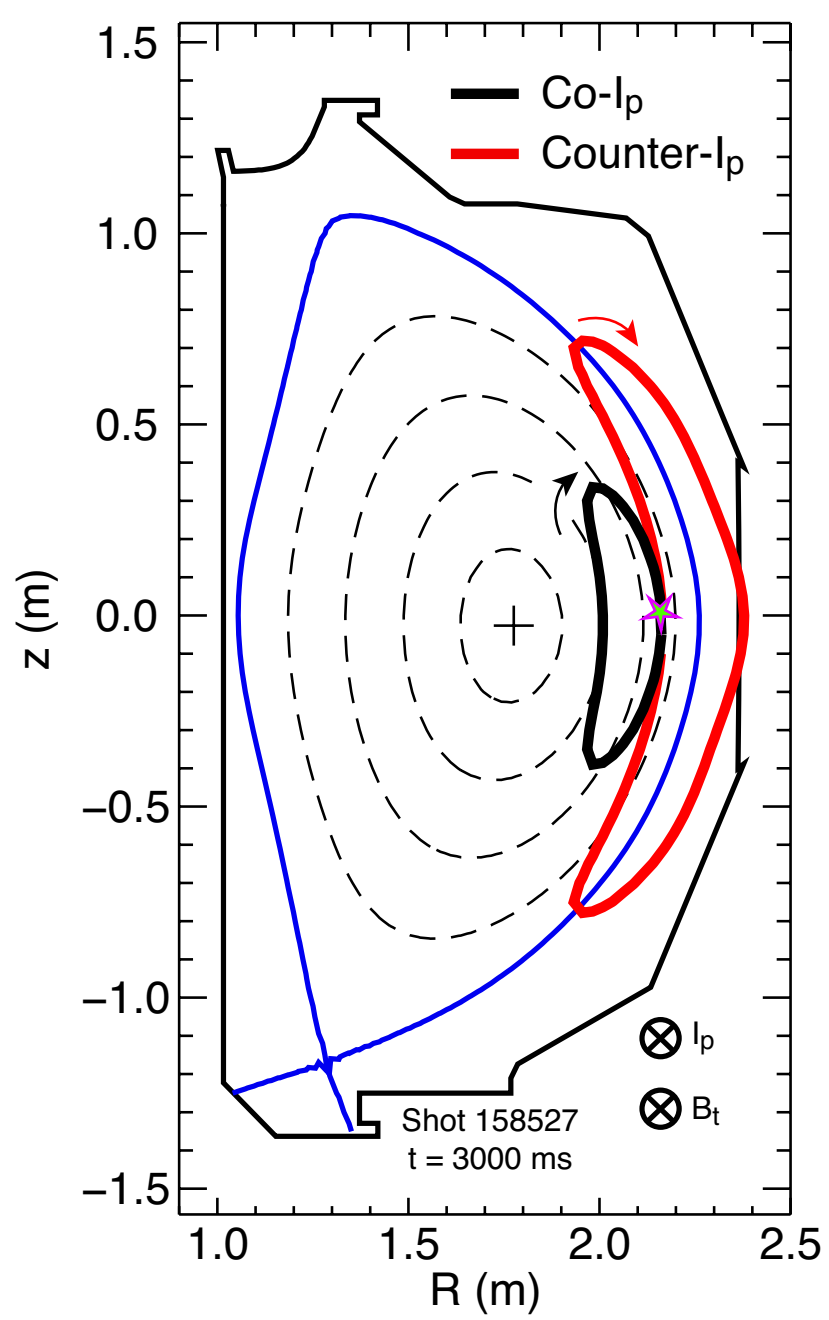

Figure 3: Example orbits from DIII-D shot 158527 at $3000 \mathrm{~ms}$. Both ions begin at the position indicated by the $\star$-symbol and feature energy $E=80 \mathrm{keV}$ and birth pitch angle (with respect to the magnetic field) $\theta=72.5^{\circ}$. One of the ions is born with a toroidal velocity vector parallel to the background plasma current (co- $I_{p}$, black trace) and the other begins with a trajectory opposite to the plasma current (counter- $I_{p}$, red trace). 


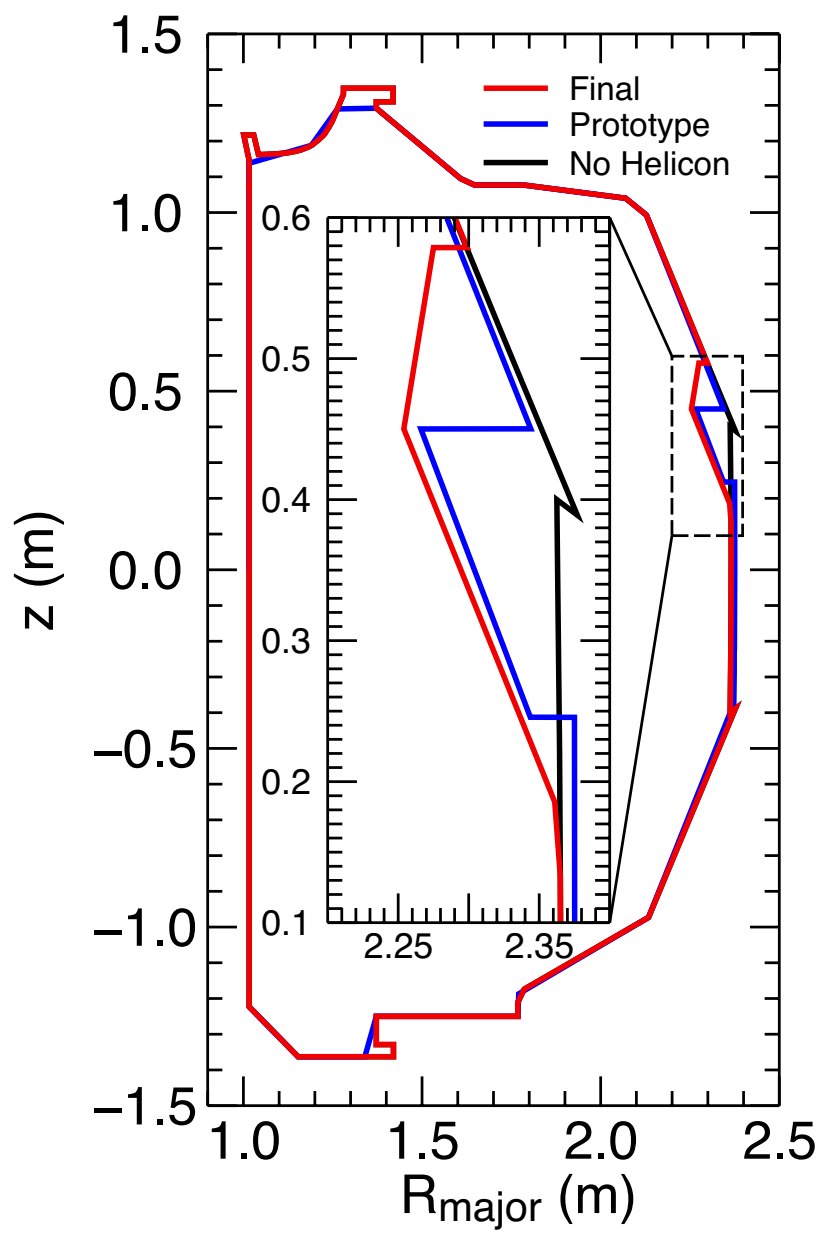

Figure 4: Different wall shapes used in the modeling including the final measured outline (red trace), the shape used for the prototype modeling (blue trace), and the standard DIII-D limiter without any antenna (black). The inset graphic is a zoomed-in view of the limiter shape only including the helicon antenna region. 

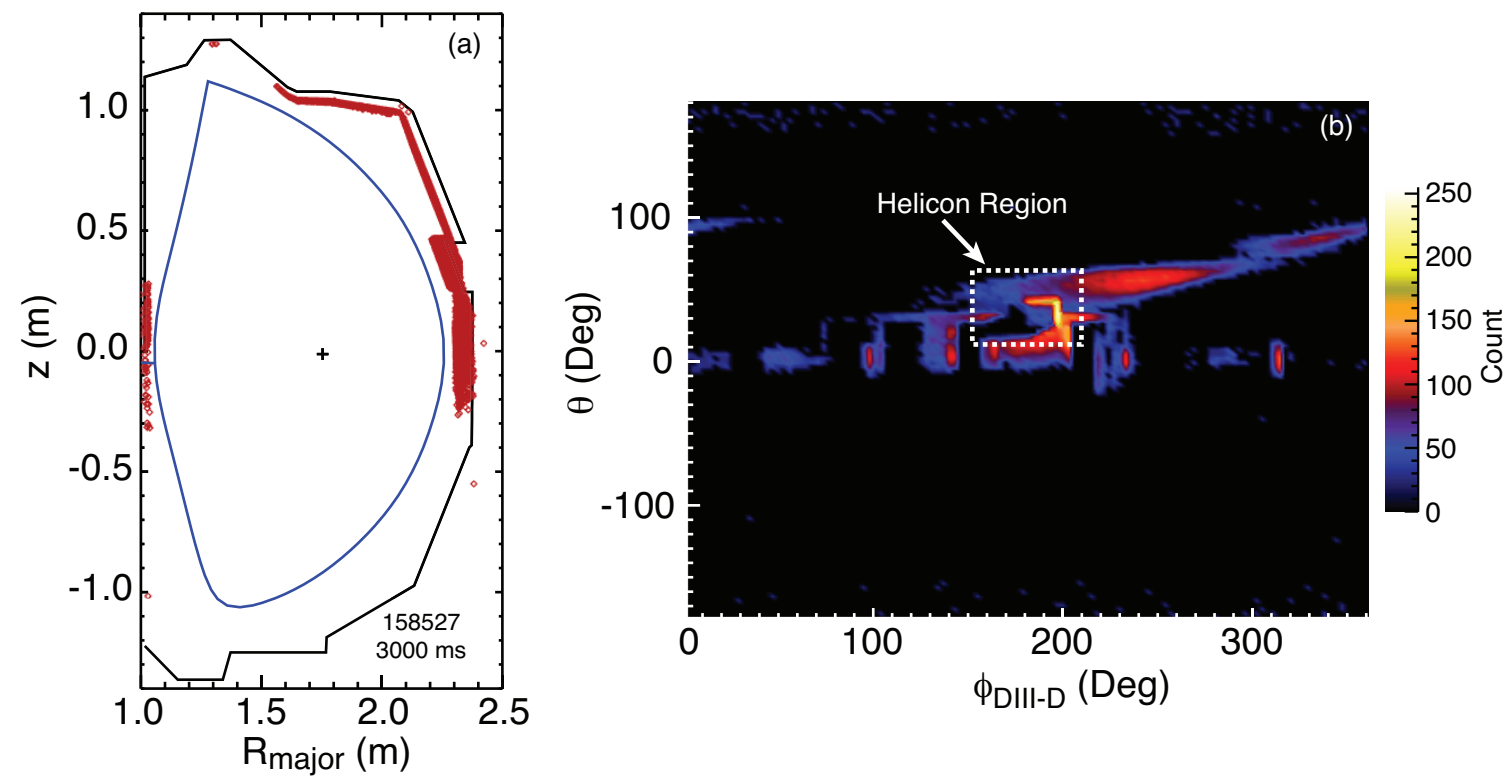

Figure 5: Modeled prompt losses from the 210L neutral beam in shot 158527 at $t=3000 \mathrm{~ms}$.

(a) $R z$-projection indicating the locations (red $\diamond$-symbols) where injected beam ions reach the outer wall for the case of the prototype only (without protective tiles, see blue trace in Fig. 4). (b) Projection of prompt losses in the $\phi_{\text {DIII-D }} \theta$-plane. 


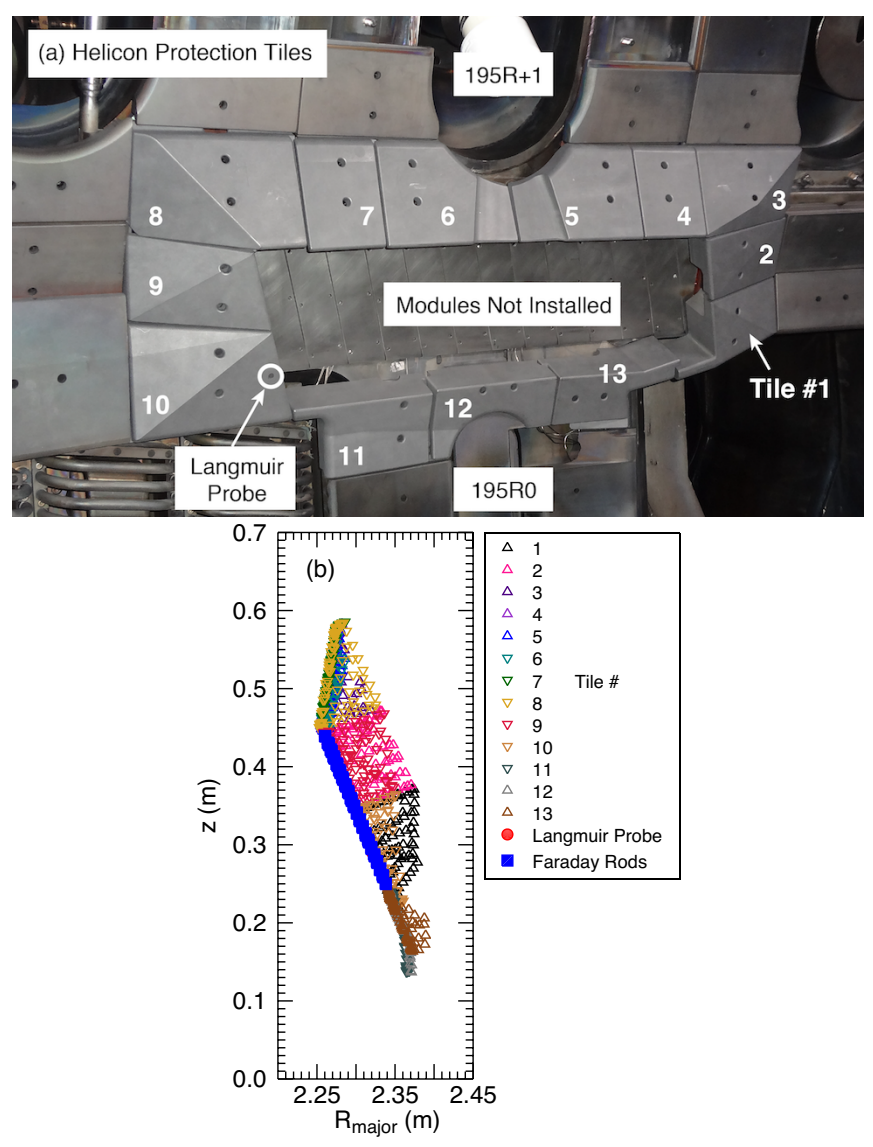

Figure 6: (a) Photograph of the helicon antenna protection tiles with identifying number tags. (b) All $\left(R_{\text {major }}, z\right)$ values for the antenna position measurement set. 

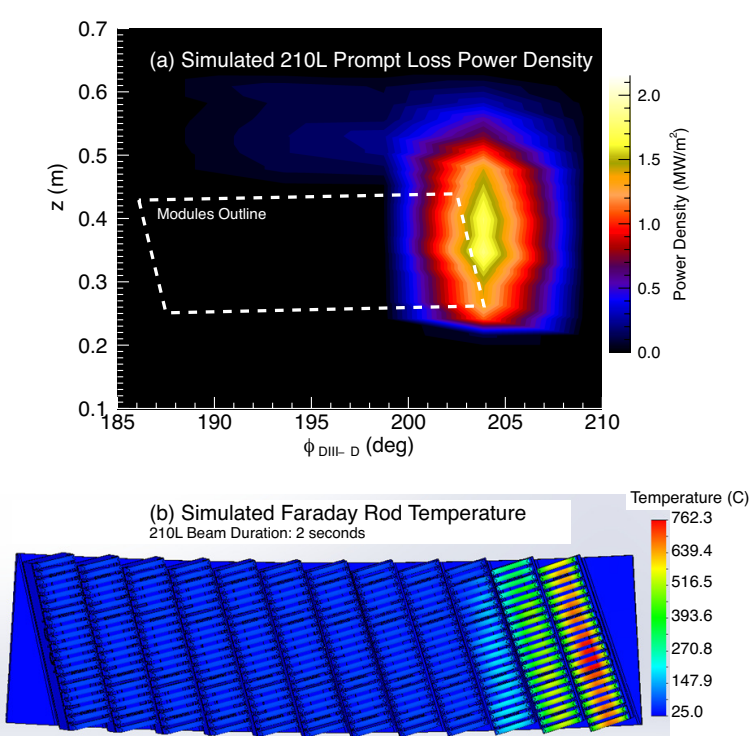

Figure 7: (a) Simulated power density across the helicon antenna installation due to prompt losses from the 210L neutral beam in shot 164468 at $t=3401 \mathrm{~ms}$. The dashed white line is an outline of the measured location of the helicon modules. (b) Simulated temperature of the helicon Faraday rods after two seconds of sustained incident power as shown in panel (a). 


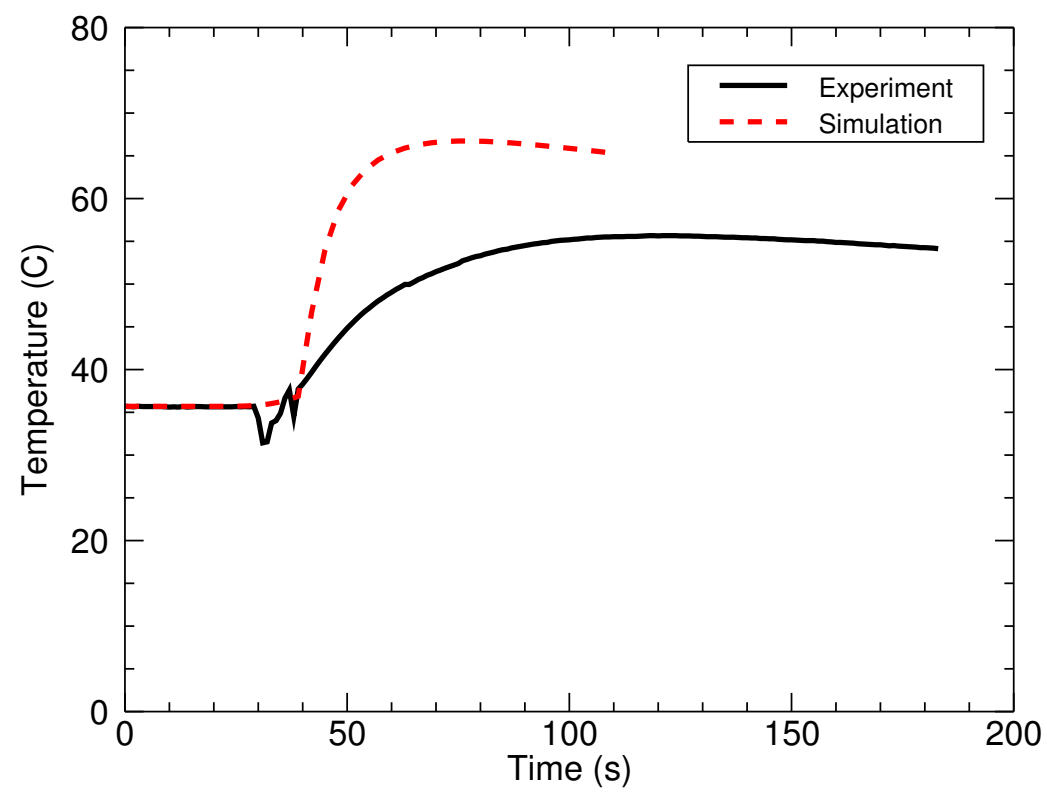

Figure 8: Temporal evolution of the measured (solid black trace) and simulated (dashed red trace) temperature of thermocouple 7 during shot 164468 (arbitrary time offset). 


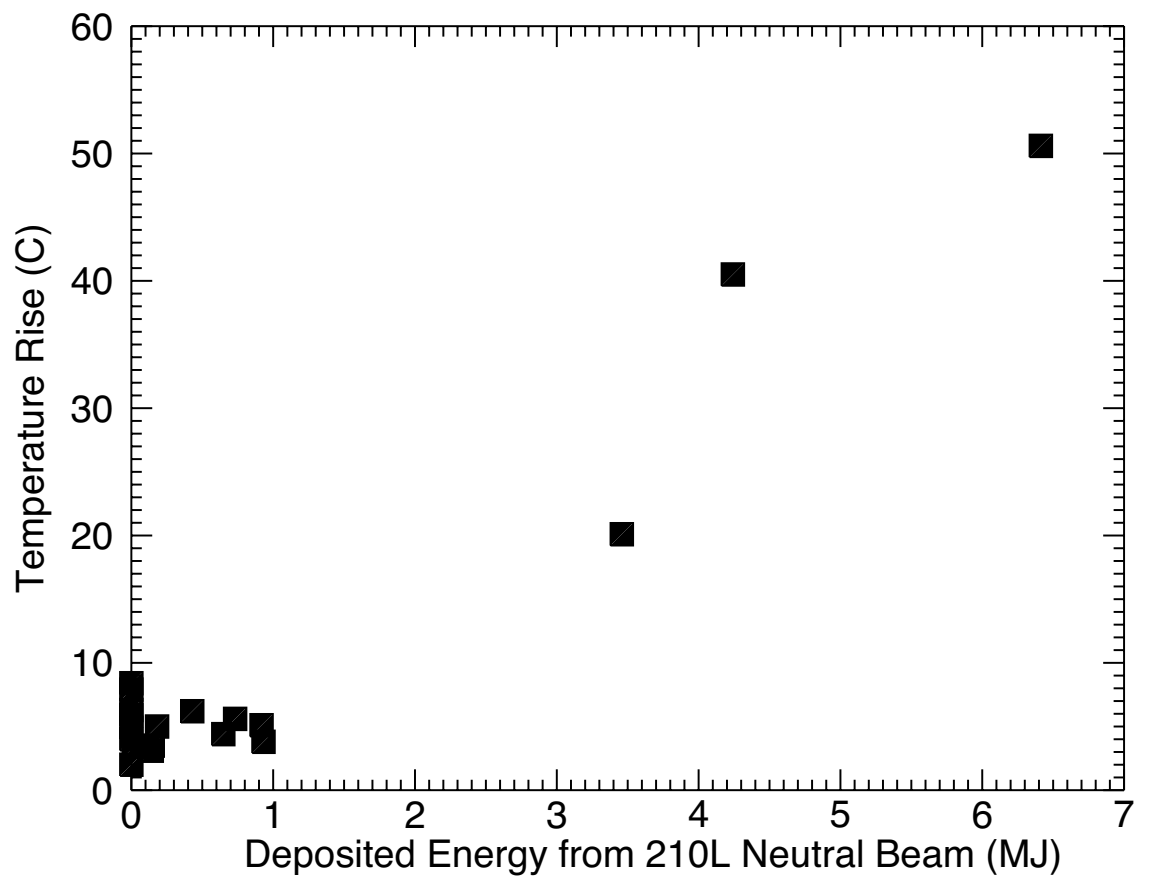

Figure 9: The change in temperature measured by thermocouple \#7 as a function of the total energy injected into the plasma by the 210L neutral beam. 


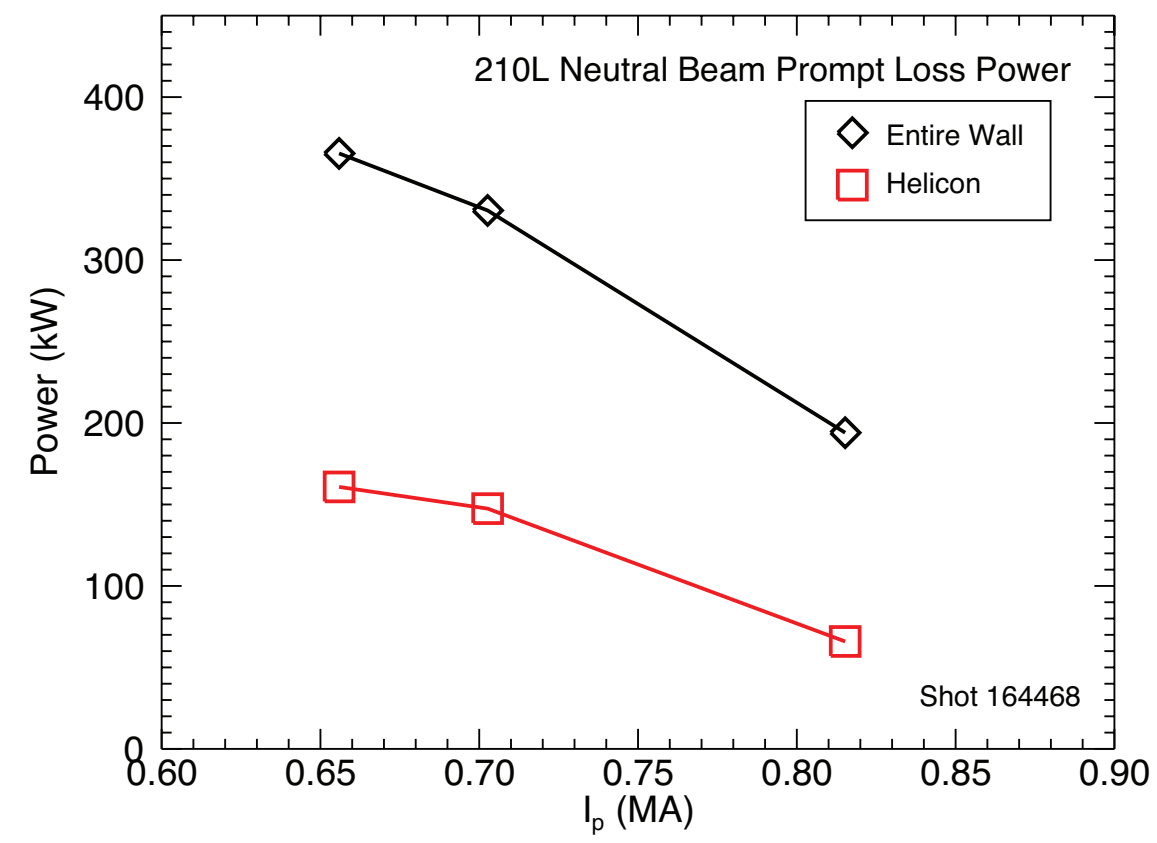

Figure 10: Simulated prompt loss power from the 210L neutral beam in shot 164468. The power is shown as the total reaching the wall (diamond shapes with black trace) and only the power reaching the helicon antenna (square shapes with red trace). 
Simulated Faraday Rod Temperature Following $2 \mathrm{~s}$ of Neutral Beam Prompt Loss
Temperature (C) 762.3

639.4

516.5

393.6

270.8

147.9

25.0 